\title{
Les morts encombrants. Du gouvernement politique des cadavres
}

Introduction

Milena Jakšić et Nicolas Fischer

\section{CpenEdition}

Journals

\section{Édition électronique}

URL : https://journals.openedition.org/conflits/22549

DOI : $10.4000 /$ conflits. 22549

ISSN : $1777-5345$

Éditeur :

CECLS - Centre d'études sur les conflits - Liberté et sécurité, L'Harmattan

Édition imprimée

Date de publication : 25 juillet 2021

Pagination : 7-14

ISBN : 978-2-343-23898-2

ISSN : 1157-996X

Référence électronique

Milena Jakšić et Nicolas Fischer, «Les morts encombrants. Du gouvernement politique des

cadavres », Cultures \& Conflits [En ligne], 121 | Printemps 2021, mis en ligne le 25 juillet 2021, consulté le 02 août 2021. URL : http://journals.openedition.org/conflits/22549 ; DOI : https://doi.org/10.4000/ conflits. 22549 


\section{Les morts encombrants. Du gouvernement politique des cadavres}

Introduction

\section{Milena JAKSIC, Nicolas FISCHER}

Milena Jakšić est sociologue, chargée de recherche au CNRS à l'Institut des sciences sociales du politique (ISP, Université Paris Onest Nanterre La Défense, ENS Paris-Saclay). Après avoir travaillé sur les politiques de lutte contre la traite des êtres humains en France, ses recherches actuelles portent sur le traitement judiciaire de l'enfance en guerre par les tribunaux pénaux internationaux. Elle a notamment publié: La traite des êtres humains en France. De la victime idéale à la victime coupable, Paris, CNRS Éditions, 2016; "Trouver l'enfant soldat. L'enquête judiciaire dans l'affaire Thomas Lubanga Dyilo", Terrain, mis en ligne le 6 juin 2019.

Nicolas Fischer est chargé de recherche CNRS en science politique au Centre de recherche sociologique sur le droit et les institutions pénales, et coordinateur du projet $A N R$ "Les corps témoins. Pour une sociologie politique du traitement des restes bumains" (CORTEM). Ses travaux antérieurs consacrés à l'enfermement des étrangers et au contrôle démocratique des lieux d'enfermement l'ont amené à développer une analyse sur l'exercice de la force publique en démocratie, et sa confrontation avec l'impératif démocratique de respect des droits des personnes. Il a notamment publié Le territoire de l'expulsion. La rétention administrative des étrangers et l'État de droit en France, Lyon, ENS Éditions, 2017.

$\mathrm{L}$ es recherches sociologiques et anthropologiques prenant la mort pour objet se sont en grande partie constituées par opposition à la thèse du " déni de la mort » popularisée en France par Philippe Ariès et Louis-Vincent Thomas ${ }^{1}$. Loin de circonscrire le destin des morts au déni, et encore moins à l'inexistence, au néant et à l'inaction, le renouveau des travaux sur la mort se propose au contraire d'explorer la multitude des relations tissées entre morts

1. Voir Ariès P., L'homme devant la mort, Paris, Seuil, 1977, et Thomas L.-V., Anthropologie de la mort, Paris, Payot, 1975. Ces travaux, très discutés, concluaient à une occultation contemporaine de la mort, qui s'opérait jadis à domicile et au cœur de la vie sociale, mais qui se trouverait aujourd'hui cantonnée à des espaces spécialisés et fermés qui la soustraient à l'expérience ordinaire. 
et vivants ${ }^{2}$, entre « ceux qui restent »- mais qui donnent ainsi aux morts un "plus d'existence » - et « ceux qui ne partent pas tout à fait » et interfèrent ainsi « dans la vie des vivants ${ }^{3}$ ».

Ce dossier de Cultures $\mathcal{E}$ Conflits s'intéresse lui aussi aux différents «modes d'existence » des morts. Mais, dans le prolongement de quelques études plus récentes, il s'intéresse non pas aux morts en général ou à « la mort » entendue comme événement ou comme processus, mais au cadavre c'est-à-dire à ce que la mort laisse derrière elle : une dépouille dont les vivants doivent disposer d'une manière ou d'une autre. À partir de cinq études de cas ${ }^{4}$, c'est donc à ces restes qu'il s'agit de s'intéresser, en les décrivant comme des objets dont la gestion particulière dépend de leurs caractéristiques inhérentes ou supposées, mais aussi et surtout des contextes sociaux dans lesquels ils sont saisis et qui façonnent leur traitement social ${ }^{5}$. Le présent dossier se concentre sur la dimension politique d'un tel traitement, soit le moment où les corps morts dont on ne peut disposer deviennent problématiques au point de susciter l'intervention des pouvoirs publics, historiquement chargés de la gestion des cadavres, mais ici interpellés par des acteurs non étatiques, familles des défunts ou membres d'organisations militantes.

Notre approche s'inscrit dans le regain d'intérêt scientifique et public qu'a connu ces dernières années la question des corps morts dans leur matérialité, et des différents traitements ou usages dont ils font l'objet. À l'échelle globale, le développement rapide de la justice pénale internationale depuis le début des années 2000 a mis en visibilité les cadavres, souvent « en masse », que laissent derrière eux les régimes autoritaires et les violences extrêmes de tous ordres. La recherche et l'exhumation de ces corps viennent appuyer un objectif de commémoration, mais aussi des procédures judiciaires : les cadavres, leur état et leur emplacement servent de preuve lorsqu'il s'agit de juger les auteurs présumés des massacres ${ }^{6}$. C'est en lien avec cette évolution que l'anthropologie physique a connu lors de la même décennie un forensic turn que les sciences sociales ont à leur tour analysé, jusqu'à en proposer une description aujourd'hui particulièrement riche ${ }^{7}$. Un autre ensemble d'études centrées sur les cadavres et leur devenir examine, à l'échelle des États-nations, les corps

2. Voir Esquerre A., Les os, les cendres et l'État, Paris, Fayard, 2011, et Molinié M., Soigner les morts pour guérir les vivants, Paris, Seuil, 2006.

3. Despret V., Au bonheur des morts, Paris, La Découverte, 2017.

4. Ce dossier est issu d'une Section thématique organisée en juillet 2019 lors du XVe Congrès de l'Association française de science politique et adossée au programme ANR CORTEM («Les corps-témoins. Pour une sociologie politique du traitement des restes humains »). Pour des précisions sur ce programme voir https://cortem.hypotheses.org/.

5. Esquerre A., Truc G., « Morts et fragments de corps ", Raisons politiques, n41, 2011, pp. 511.

6. Anstett É., Des cadavres en masse. Sociétés et sciences sociales face à l'impensé, Paris, Éditions de la Maison des sciences de l'homme, 2013 ; Claverie É., « Réapparaître. Retrouver les corps des personnes disparues pendant la guerre en Bosnie », Raisons politiques, $\mathrm{n}^{\circ} 41,2011$, pp. 13 31 . 
dont le statut, les conditions de leur disparition et bien souvent le nombre empêchent de "traiter » selon les procédures normales d'enregistrement et d'inhumation - ce qui fait de leur présence un enjeu médiatique pris en charge avec plus ou moins d'improvisation par les pouvoirs publics. C'est le cas par excellence pour les victimes d'attaques terroristes ${ }^{8}$, ou encore de catastrophes naturelles ou sanitaires, de la canicule qui a frappé la France en 2003 à la pandémie de Covid-19 qu'elle traverse actuellement 9 .

Les cas concrets décrits dans ce dossier relèvent de quelques unes de ces situations : il peut s'agir de traiter les corps des victimes de tueries de masse perpétrées par des organisations politiques (Dorothée Delacroix) ou criminelles (Sabrina Melenotte); de se confronter aux cadavres présents ou manquants des terroristes (Lisa Carayon, Antoine Mégie); ou enfin de magnifier ou d'occulter des sépultures selon le rôle qu'elles jouent dans la construction d'un récit national (Thomas Richard).

Ces cadavres encombrants ont tout d'abord en commun leur caractère chaque fois problématique. Comme le remarquaient il y a quelques années Arnaud Esquerre et Gérôme Truc à propos d'autres corps ${ }^{10}$, ils se caractérisent également, en premier lieu, par leur caractère déplacé, au propre comme au figuré. Le regard sociologique qui les prend pour objet doit alors être particulièrement attentif aux lieux dans lesquels ils reposent ou devraient reposer, autant qu'à la manière dont on les fait circuler d'un espace à l'autre. De ce point de vue, les corps étudiés dans ce dossier relèvent de lieux, de déplacements et donc de modes d'existence très divers : nous évoquerons des mortsabsents ou introuvables d'auteurs d'actes de terrorisme islamiste (Antoine Mégie), ou ceux qui, au contraire, sont trouvés et exécutés, mais à qui on refuse une sépulture tant leur mort dérange et leurs corps sont objet de haine (Lisa Carayon). D'autres morts apparaissent sous forme de "déchets jetables » retrouvés mais pas toujours identifiés dans des « espaces clandestins d'exécution » au Mexique (Sabrina Melenotte). En allant plus au sud, au Pérou, nous assistons à d'autres pratiques de manipulation des cadavres en contexte post-conflit. Nous y observons notamment des pratiques de déterrements des restes humains par l'État péruvien, trente ans après la guerre civile,

7. Parmi une production abondante, voir notamment Dziuban Z., Mapping the 'forensic turn': engagements with materialities of mass death in Holocaust studies and beyond, Vienne, New academic press, 2017 ; également Ferrándiz, F., Robben A. (eds.), Necropolitics: mass graves and exhumations in the age of human rights, Philadelphia, University of Pennsylvania Press, 2015. Pour une synthèse de ces travaux, voir Le Clainche-Piel, M., "État de littérature. Du public à l'intime : le traitement des cadavres problématiques vu par les sciences sociales ", Critique internationale, vol. 87, n², 2020 pp. 201-216.

8. Aronson J.-D., Who owns the dead? The science and politics of death at Ground Zero, Cambridge, Harvard University Press, 2016, et Truc G., Sidérations : une sociologie des attentats, Paris, Presses Universitaires de France, 2016.

9. Clavandier G., La mort collective. Pour une sociologie des catastrophes, Paris, CNRS Éditions, 2004.

10. Esquerre A., Truc G., « Morts et fragments de corps », op. cit. 
pour traquer les familles soupçonnées de fraude au dispositif de réparation (Dorothée Delacroix). Celles-ci accusent à leur tour l'État de transformer les squelettes de leurs proches en exhausteurs de goût pour l'industrie alimentaire. En contraste avec ces corps manipulés ou transformés en déchets, on parlera des morts sacralisés à Jérusalem, donnés à voir aux touristes selon un itinéraire urbain soigneusement délimité, caractérisé cependant par la tendance à délaisser, voire à dissimuler aux regards les morts palestiniens qui cadrent mal avec le récit national en vigueur (Thomas Richard).

On le comprend à la lecture de ce panorama, les enjeux de localisation des corps devenus cadavres sont d'emblée des enjeux politiques, et ils contribuent à la politisation du traitement des corps dont il est question ici. Le second trait commun des corps analysés est bien, de fait, leur capacité à problématiser l'État-nation qui s'est voulu le contrôleur ultime du traitement des corps morts depuis le XIX ${ }^{\text {e }}$ siècle ${ }^{11}$. Parmi les cadavres qui viennent d'être évoqués, ceux des terroristes sont ainsi marqués par une allégeance à un ensemble plus vaste que l'État-nation ${ }^{12}$ et s'en excluent au point que des maires se refusent à les inhumer sur le territoire de leur commune (L. Carayon), ou que d'autres responsables étatiques cherchent en vain à confirmer leur mort en Syrie (A. Mégie). D’autres corps sont délibérément abandonnés par les autorités étatiques (S. Melenotte), ou sont au contraire saisis pour mieux être « oubliés », voire égarés (D. Delacroix), lorsque leurs sépultures ne sont pas physiquement mises à l'écart de la communauté nationale (T. Richard). De ces diverses situations de rejet au moins partiel des corps par l'État-nation, témoignent les vocables et idiomes utilisés pour désigner les corps. Le vocabulaire dépréciatif domine : il est ainsi question de "présumés morts » (A. Mégie), de « morts qui dérangent ", de « corps haïs » (L. Carayon), de « sujets négligés », de «bonne poubelle» (S. Melenotte), de «squelettes transformés », de « cadavres-déchets », de « biens aliénables » (D. Delacroix), ou « des sépultures spoliées » (S. Melenotte, D. Delacroix, T. Richard). La récurrence de telles caractérisations inscrit les corps dont il est question ici dans la continuité des « corps vils »- ceux des indigents, des vagabonds, des prisonniers ou des condamnés exécutés, dont la marginalisation de leur vivant se prolonge également post mortem : jugée indigne du moindre égard, leur dépouille est jetée à la fosse commune, ou fréquemment disséquée dans les Facultés de médecine ${ }^{13}$. Elle confirme qu'ils sont aujourd'hui, au mieux, des corps « problématiques » qui dérangent, encombrent, embarrassent, font l'objet de suppliques ou de contestations.

11. Esquerre A., Les os, les cendres et l'État, op. cit.

12. Kastoryano R., Que faire des corps des djihadistes? Territoire et identité, Paris, Fayard, 2015.

13. Chamayou G., Les corps vils : expérimenter sur les êtres humains auX XVIII et XIXe siècles, Paris, La Découverte, 2014. Sur les usages médicaux des corps des indigents et des condamnés, voir par exemple Rossigneux-Méheust M., « Négocier sa mort. Le combat des vieillards en institution à Paris au XIX ${ }^{\mathrm{e}}$ siècle ", Revue d'histoire moderne E contemporaine, vol. 61, n³, 2014, pp. 98-123, et Ward R. (ed.), A Global History of Execution and the Criminal Corpse, New York, Palgrave Macmillan, 2015. 
Il faut toutefois le noter, les cinq cas étudiés ici se distinguent des morts que laissent derrière eux d'autres situations extrêmes - catastrophes naturelles, attentats ou crimes de masse - par une dimension supplémentaire : leur politisation est inachevée. Elle reste cantonnée à une arène d'acteurs restreinte, et l'investissement de certains protagonistes - notamment les représentants des États - dans le traitement des corps y est lui-même limité ${ }^{14}$. La singularité des cas réunis dans ce dossier réside alors précisément dans le silence qui entoure les corps : ceux-ci existent en grand nombre mais souffrent d'un déficit de visibilité. Honnis, absents ou négligés, ils suscitent peu de controverses, ou bien les débats qui les concernent demeurent confinés dans une arène bien délimitée. On ne trouvera donc pas ici les controverses ou les disputes entre acteurs étatiques et non étatiques, entre savoirs experts et savoirs profanes, auxquelles on aurait pu s'attendre. Contrairement aux enquêtes pour crimes de guerre, les cadavres visés dans le présent dossier font rarement l'objet d'enquêtes judiciaires fouillées, et les procès, lorsqu'ils ont lieu, ne s'internationalisent pas, ne suscitent aucun scandale et deviennent encore moins des affaires ${ }^{15}$.

C'est le silence socialement et politiquement organisé qui constitue donc le point de convergence entre les différents papiers. Qu'on ne s'y méprenne pas : ce silence a ses propres modalités d'énonciation, et il n'est pas toujours le résultat d'une action délibérée ou d'une stratégie clairement définie. Il relève davantage de pesanteurs bureaucratiques, de négligences administratives, du sentiment d'indifférence et de lassitude, ou du manque de ressources sociales des familles qui peinent à désingulariser leur cas pour en faire une cause susceptible d'intéresser le plus grand nombre. On parle bien de ces morts, mais les situations d'énonciation dans lesquelles ils sont pris empêchent toute publicisation de cette parole. La médiatisation elle-même, lorsqu'elle a lieu, sert « à faire taire 16 » les responsabilités individuelles ou institutionnelles.

De cette organisation sociale du silence, il est d'abord question dans la contribution d'Antoine Mégie. Enquêtant pendant cinq ans sur les procès de violence terroriste, l'auteur choisit de centrer son regard sur une nouvelle catégorie des procès criminels, celle des «présumés morts » en Irak et en Syrie. Ce vocable désigne des personnes poursuivies en France pour des actes terroristes, mais dont les corps demeurent introuvables. Jugés par défaut, ces

14. On se fonde ici sur une approche classique de la politisation comme activité collective supposant avant tout un travail de montée en généralité : une situation de départ est identifiée comme conflictuelle par des acteurs qui en imputent la responsabilité à un adversaire, une institution étant in fine sollicitée pour la traiter. Sur ce point, Hamidi C., La société civile dans les cités. Engagement associatif et politisation dans des associations de quartier, Paris, Économica, 2010.

15. Offenstadt N., Claverie É., Boltanski L., Van Damme S. (dir.), Affaires, scandales et grandes causes. De Socrate à Pinochet, Paris, Stock, 2007.

16. Voir notamment Léonore Le Caisne à propos de la mise en scène médiatique de l'inceste : Le Caisne L., Un inceste ordinaire. Et pourtant tout le monde savait, Paris, Belin, 2014. 
«morts invérifiables " font l'objet d'un traitement judiciaire particulier. Contrairement au procès des attentats de Charlie Hebdo et de l'Hyper Cacher de la Porte de Vincennes jugés devant une cour d'assises à Paris, entièrement enregistrés et filmés en raison de leur «portée historique », les audiences des «présumés morts » étudiées par Antoine Mégie peinent à se faire entendre. Leur traitement relève toutefois d'une procédure inédite, celle du « circuit court », fondé sur le principe de comparutions immédiates. Le but est d'accélérer la procédure, d'aller vite tant l'existence même de cette nouvelle figure de l'accusé dérange. L'État veut en effet absolument juger un mort dont le corps absent ne doit surtout pas revenir. Il ressort de l'ethnographie menée par Antoine Mégie une scène judiciaire silencieuse jusqu'au mutisme. Les audiences ne sont suivies par aucun public, hormis l'ethnographe, quelques touristes curieux ou égarés, et les proches de l'accusé. L’absence du «présumé mort » du box des prévenus, doublée de l'absence de ses avocats, empêche tout débat contradictoire. Les présidentes et les présidents d'audiences font de longs monologues monotones lors des séances qui dépassent rarement une demi-heure. Cette rapidité de la procédure s'explique par la difficulté à recueillir des preuves sur «les personnes qui n'ont pas pu être arrêtées » et dont les corps restent introuvables.

La contribution de Lisa Carayon offre un exemple inverse. Les corps des auteurs d'actes de terrorisme islamiste sont trouvés et identifiés, mais c'est le lieu de leurs funérailles qui suscite crainte et inquiétude. L'auteure assiste ainsi à un enterrement en secret, de nuit, le corps de celui désigné comme « ennemi de la France » étant enterré dans une tombe qui ne portera pas son nom. Le cimetière devient ici un véritable «terrain de conflit ». Faut-il accepter les funérailles publiques de ces morts qui dérangent ou au contraire les exclure du territoire national ? Si les maires qui ont accepté l'inhumation affirment l'avoir fait par respect du principe républicain de l'égalité des citoyens devant la mort, d'autres ont justifié leur refus par crainte de troubles à l'ordre public ou par solidarité avec les victimes d'actes terroristes. En croisant l'analyse du droit funéraire français aux entretiens réalisés avec les maires responsables des funérailles, Lisa Carayon nous invite à réfléchir aux multiples usages pénaux du corps de l'ennemi ou du coupable. Se donne ici à voir une gestion différenciée des corps selon quelques grands partages qui opposent les morts socialement pertinents, que les familles mais aussi les services funéraires publics se doivent de prendre en charge, et les corps faisant l'objet d'un traitement minimal, voire inexistant. Les corps des auteurs d'actes de terrorisme n'entrent pas dans la catégorie des morts illustres ou socialement respectables. Leur traitement - en silence, de nuit, hors du carré musulman, voire dans une partie inutilisée du cimetière résonne avec le traitement des cadavres des populations déjà marginalisées de leur vivant. 
Les contributions d'Antoine Mégie et de Lisa Carayon nous éclairent ainsi sur les morts dont les modes d'existence posent problème à l'Étatnation : l'État peut refuser de prendre en compte un corps qui s'est exclu luimême (L. Carayon), ou bien vouloir absolument juger un mort dont le corps absent ne doit surtout pas revenir (A. Mégie).

À côté de ces « morts infâmes », les deux articles suivants explorent un autre mode d'existence des morts, celui des «corps-déchets » et de leur manipulation par une série d'acteurs institutionnels et non-institutionnels. Au Mexique, Sabrina Melenotte nous emmène sur les traces des « espaces clandestins de la disparition » afin d'y interroger « les frontières de la mort » causée par les activités criminelles des cartels. Alors que le pays est devenu une " immense fosse commune ", les 300000 morts recensés au Mexique ont leur géographie propre. Nous les retrouvons dans les décharges, dans les fosses clandestines, au bord des routes, dans les camions frigorifiques, très souvent à l'état de cendres pour empêcher toute identification. Sabrina Melenotte part sur leurs traces en suivant des collectifs de recherche des personnes disparues. Composés de familles qui, " poussées par la douleur », ont appris «à lire la terre » et d'experts médico-légaux issus d'organisations non-gouvernementales, ces collectifs hybrides se caractérisent par une méfiance généralisée visà-vis de l'État et de ses institutions. Ils savent que la réduction de leurs proches en déchets sert à camoufler les responsabilités - y compris étatiques dans les violences perpétrées. Sabrina Melenotte pointe ainsi une tension fondamentale au sein de ce qu'elle désigne comme le «nécropouvoir » mexicain. Cette tension oppose d'un côté les familles, qui ont «besoin de matérialité dans leur quête de vérité ", et de l'autre l'État, qui manipule les corps jusqu'à les faire disparaître, afin de dissimuler les informations sur les cadavres, voire sa propre responsabilité.

Une configuration sensiblement différente est observée par Dorothée Delacroix au Pérou. Ici, les corps des victimes de la guerre civile sont bel et bien identifiés et enterrés, mais se voient exhumés trente ans après les faits afin de « vérifier l'exactitude des témoignages des survivants » suspectés de fraude au dispositif de réparation. Cette traque aux « bénéficiaires indus » donne lieu à des pratiques de manipulation des restes humains vécues comme de véritables « dérangements posthumes » par les familles. Placés dans des boîtes à gâteaux, ils sont transportés vers des destinations inconnues afin d'y être à nouveau examinés. Les familles qui attendent parfois plus de trois ans la restitution des fragments des corps, accusent à leur tour l'État d'utiliser les restes de leurs proches pour la fabrication d'exhausteurs de goût. Cette rumeur largement répandue fait basculer les morts étudiés par Dorothée Delacroix du statut de « personne » à celui de «fragments d'os commercialisé ». 
La cinquième contribution, par Thomas Richard, travaille d'une autre manière la thématique de visibilité ou au contraire d'invisibilité conférée à certains morts. Dans son enquête sur le marquage territorial de la ville de Jérusalem, l'auteur décrit comment l'État d'Israël, par sa politique de recomposition du paysage funéraire, est parvenu à construire une relation intime entre les visiteurs et les défunts et à procéder à une « articulation très étroite entre les sépultures antiques et [...] l'État contemporain ». Les diverses dimensions du sacré sont ici mobilisées à des fins politiques. Elles constituent des objets de mémorialisation et une ressource précieuse pour le nationalisme étatique prompt à exploiter les dimensions de la terre et des morts. Mais cette légitimation de la présence judéo-chrétienne implique l'invisibilisation des sites funéraires palestiniens qui mettent à mal le récit national en vigueur. La mise en visibilité de certains sites implique nécessairement la dissimulation des morts qui dérangent.

C'est donc à une analyse de ces diverses manifestations du gouvernement politique des cadavres, qu'invitent les différentes contributions rassemblées dans ce numéro. Elles mettent tout d'abord en évidence la problématisation contemporaine de corps qu'on a déjà définis comme « vils »- les corps des criminels, des ennemis, des populations socialement négligeables. Cette problématisation n'est en elle-même pas nouvelle, le mépris traditionnellement témoigné à ces cadavres ayant déjà fait l'objet de critiques par le passé ${ }^{17}$. Bien que les disputes présentées ici restent le plus souvent des disputes avortées, qui ne permettent dès lors qu'une politisation partielle des cadavres et de leur traitement, leur essor témoigne de l'apparition de nouveaux formats de discussion autour des corps. Comme nous l'avons indiqué, la variété des situations analysées souligne alors l'importance des échelles d'action des protagonistes du travail de politisation : au fil des contributions, l'enjeu est de rendre visible les corps et leur traitement indigne ou leur négligence, pour dépasser l'échelon local et interpeller les autorités étatiques, voire supra-étatiques. Elle montre aussi et surtout l'importance, dans ce travail, des experts officiels ou improvisés : qu'il s'agisse des médecins légistes agréés ou de détenteurs d'un savoir local permettant par exemple d'identifier les morts ou de reconstituer leur histoire, ils jouent un rôle crucial de passeurs, non seulement entre les vivants et les cadavres qu'ils analysent et «font parler », mais aussi entre les différents espaces sociaux dans lesquels ce que les corps ont à dire peut être entendu, et peut produire ou non des effets politiques.

17. Mathilde Rossigneux-Méheust montre par exemple comment la dissection puis la destruction des corps des indigents morts dans les hospices parisiens a suscité des mobilisations et l'indignation de la presse, dès la fin du XIXe siècle. Voir Rossigneux-Méheust M., "Négocier sa mort. Le combat des vieillards en institution à Paris au XIXe siècle ", op. cit. 